\title{
The Impact of High Pressure, Doping and the Size of Crystalline Boron Grains on Creation of High-Field Pinning Centers in In Situ MgB $\mathbf{B}_{2}$ Wires
}

\author{
D. Majchrzak ${ }^{1}$ (D) A. Zaleski ${ }^{2}$ - A. Morawski ${ }^{3} \cdot$ M. Małecka ${ }^{2}$ - M. Rindfleisch ${ }^{4} \cdot$ D. Gajda ${ }^{2}$
}

Received: 21 June 2018 / Accepted: 27 June 2018 / Published online: 6 July 2018

(C) The Author(s) 2018

\begin{abstract}
In this work, we investigated the influence of isostatic pressure (of $0.1 \mathrm{MPa}$ and $1.1 \mathrm{GPa}$ ) during heat treatment, doping, size and shape of crystalline boron grains, and annealing time at low annealing temperature $\left(570{ }^{\circ} \mathrm{C}\right)$ on the formation of highfield pinning centers in $\mathrm{MgB}_{2}$ wires. The results indicate that high isostatic pressure (1.1 GPa) at low annealing temperature $\left(570{ }^{\circ} \mathrm{C}\right)$ and annealing time of 120 min significantly increases the density of high-field pinning centers in $\mathrm{MgB}_{2}$ wires with small and large boron grains. Transport measurements show that an increase of annealing time from 120 to $210 \mathrm{~min}$ at $1.1 \mathrm{GPa}$ slightly decreases the critical current density $\left(J_{\mathrm{c}}\right)$ and irreversibility field $\left(B_{\text {irr }}\right)$ in $\mathrm{MgB}_{2}$ wires with large boron grains, suggesting that longer annealing time weakly affects the density of high-field pinning centers. On the other hand, for $\mathrm{MgB}_{2}$ wires with small boron grains, the annealing time of $210 \mathrm{~min}$ significantly reduces $J_{\mathrm{c}}$ and $B_{\text {irr. }}$. This indicates that the longer annealing time at $1.1 \mathrm{GPa}$ significantly reduces the density of high-field pinning centers. Our studies indicate that dislocations created by the hot isostatic pressure process significantly increase $B_{\text {irr }}$ in the temperature range from 5 to $12 \mathrm{~K}$. On the other hand, strains due to the shrinkage of $\mathrm{MgB}_{2}$ material increase $B_{\text {irr }}$ in the temperature range from 12 to $34 \mathrm{~K}$. The results show that small grains of crystalline boron and high isostatic pressure lead to a high density of dislocations and strains during transformation to the $\mathrm{MgB}_{2}$ phase.
\end{abstract}

Keywords HIP process $\cdot \mathrm{C}$ doping $\cdot$ Crystalline boron $\cdot \mathrm{MgB}_{2}$ wires $\cdot$ Pinning centers

\section{Introduction}

High-field pinning centers are not easy to obtain because they are placed inside the grains and on grain boundary [1]. These pinning centers are very important for superconductor applications because they significantly increase $J_{\mathrm{c}}$ in high magnetic fields in superconducting wires. Previous results for $\mathrm{NbTi}$ wires indicate that high-field pinning centers are created by dislocations and substitution into crystal lattice

D. Majchrzak

dommaj7@wp.pl

1 Faculty of Physics and Astronomy, University of Wroclaw, Maksa Borna 9, 50-204 Wroclaw, Poland

2 Institute of Low Temperature and Structure Research, Polish Academy of Sciences, Okólna 2, 50-422 Wroclaw, Poland

3 Institute of High Pressure Physics, Polish Academy of Sciences, Sokolowska 29/37, 01-142 Warszawa, Poland

4 Hyper Tech Research, Inc., 539 Industrial Mile Rd, Columbus, OH 43228, USA in the form of strains and $\mathrm{Ti}$ precipitates inside the grains [1]. Gajda et al. have shown that dislocations in NbTi wires most effectively increase $J_{\mathrm{c}}$ in magnetic fields from 6 to $8 \mathrm{~T}$ [1]. In addition, transport measurements indicate that substitution into crystal lattice most efficiently increases $J_{\mathrm{c}}$ in magnetic fields from 8 to $9.5 \mathrm{~T}$ and $\mathrm{Ti}$ precipitates inside the grains effectively increase $J_{\mathrm{c}}$ near $B_{\text {irr }}$ [1].

Dislocations and strains in superconducting wires can be created by cold treatment (e.g., cold isostatic pressure [2], cold drawing [3]) or hot treatment (e.g., hot isostatic pressure process which creates dislocations [4]) and also by the shrinkage of the superconducting material during the synthesis reaction [5] or substitution into crystal lattice, which creates strains in either case [6]. Dislocations created by cold treatment disappear as a result of thermal processes. The thermal processes of in situ $\mathrm{MgB}_{2}$ wires are necessary to form superconducting material. This means that foregoing cold treatment in in situ $\mathrm{MgB}_{2}$ wires does not create high-field pinning centers. This indicates that only hot treatment in $\mathrm{MgB}_{2}$ wires can create high-field pinning centers. The shrinkage of in situ $\mathrm{MgB}_{2}$ in wires 
during hot treatment allows strains to develop (which only decrease $T_{\mathrm{c}}$ by $1 \mathrm{~K}$ [7]) and creates big voids [5]. In addition, these strains have a non-uniform distribution in $\mathrm{MgB}_{2}$ wires. The big voids (larger than the coherence length) reduce the number of connections between the grains and create surface pinning centers [3], which increase $J_{\mathrm{c}}$ in low magnetic fields. The dislocations and strains can be obtained in in situ $\mathrm{MgB}_{2}$ wires by substitution of $\mathrm{C}$ for $\mathrm{B}$ during heat treatment $[6,8]$. Currently, several sources of carbon dopants are used: nano-diamond, graphene, carbon nanotubes, $\mathrm{C}_{4} \mathrm{H}_{6} \mathrm{O}_{5}$, and nano-graphite. Studies indicate that the substitution of $\mathrm{C}$ for $\mathrm{B}$ at low annealing temperatures implies slow kinetic of synthesis [9]. The low annealing temperature is important because it leads to the final $\mathrm{MgB}_{2}$ material with high density and, therefore, the large number of connections between the very fine nano-metricscale grains [10]. This leads to an increase in $J_{\mathrm{c}}$ in the $\mathrm{MgB}_{2}$ wires. The high annealing temperature accelerates the substitution of $\mathrm{C}$ for $\mathrm{B}$ but creates high kinetic of reaction and much bigger grains surrounded by bigger voids, which limits the number of connections between the grains and finally leads to a reduction of $J_{\mathrm{c}}$ [11].

The DL-malic acid $\left(\mathrm{C}_{4} \mathrm{H}_{6} \mathrm{O}_{5}\right)$ as a source of carbon dopant has many advantages. The DL-malic acid melts at lower temperatures in the mixture and decomposes at temperatures below the formation temperature of $\mathrm{MgB}_{2}$ [12]. This results in production of highly reactive and fresh $\mathrm{C}$, which accelerates the formation of superconducting phase and substitution of $\mathrm{C}$ for $\mathrm{B}$. Another product that appears in the reaction is carbon monoxide (a reducing reagent), which may convert boron oxide to B [12]. Moreover, using $\mathrm{C}_{4} \mathrm{H}_{6} \mathrm{O}_{5}$ powder allows for highly uniform mixing, because it forms a coating on the B powder surfaces [13].

Previous studies show that the hot isostatic pressing (HIP) process yielded $\mathrm{MgB}_{2}$ superconducting material with smaller grains and higher density of connections between grains [14-16]. The HIP process increases the homogeneity and density of dislocations $[17,18]$. It leads to an increase in critical current density $\left(J_{\mathrm{c}}\right)$ and irreversibility field $\left(B_{\text {irr }}\right)$ [19]. Investigations presented by Adamczyk et al. for SiCdoped $\mathrm{MgB}_{2}$ wires with $\mathrm{Nb}$ barrier have shown that the HIP process results in a $J_{\mathrm{c}}$ value of $100 \mathrm{~A} / \mathrm{mm}^{2}$ at $4.2 \mathrm{~K}$ in $14 \mathrm{~T}$ [20]. Research also suggests that the HIP process can accelerate the substitution of $\mathrm{C}$ for $\mathrm{B}$ in in situ $\mathrm{MgB}_{2}$ wires $[16,19]$. It also leads to an increase in the density of strains.

In in situ $\mathrm{MgB}_{2}$ wires with amorphous boron, the reaction rate strongly depends on the size of the boron grains. Small grains of amorphous boron increase the rate of synthesis reaction and lead to an increase in $J_{\mathrm{c}}$ and $B_{\text {irr }}$ [21, 22]. Gajda et al. indicate that a high pressure of $1.1 \mathrm{GPa}$ allows for a more efficient increase in the density of high-field pinning centers in in situ $\mathrm{MgB}_{2}$ wires with nano-amorphous boron [4] than with big amorphous boron [23]. The crystalline boron has higher hardness properties than the amorphous boron. Additionally, the crystalline boron typically has a $\beta$-rhombohedral structure, which is stable even at high temperature [9]. This means it does not fully react with $\mathrm{Mg}$ powder to create an $\mathrm{MgB}_{2}$ phase. Kim et al. show that $J_{\mathrm{c}}$ in in situ $\mathrm{MgB}_{2}$ wires with nano-crystalline and big crystalline boron are the same (with the same annealing temperature and time of annealing) [9]. Currently, the crystalline boron with low purity (96\%) is ten times cheaper than the amorphous boron with high purity (99\%) [24]. From the viewpoint of cost, the improvement of $J_{\mathrm{c}}$ in $\mathrm{MgB}_{2}$ wires made with the crystalline $\mathrm{B}$ is very important.

Our results show that only high pressure at low annealing temperature and long annealing time would lead to a significantly increase in the density of high-field pinning centers (pinning centers accumulated inside the grains) in doped $\mathrm{MgB}_{2}$ made with crystalline $\mathrm{B}$.

\section{Experimental Details}

The $\mathrm{MgB}_{2}$ wires with $\mathrm{Nb}$ barrier were made at Hyper Tech Research, Inc., using a continuous tube forming and filling (CTFF) route [25]. The samples with 30 filaments, designated as samples A, B, and C in Table 1 initially comprised a crystalline B (97\% purity; Tangshan [9]), pre-doped with 5 at. $\% \mathrm{C}_{4} \mathrm{H}_{6} \mathrm{O}_{5}$, and mixed with $\mathrm{Mg}$ powder, with an Mg-to-B ratio of 1:2. The samples with 36 filaments samples $\mathrm{D}, \mathrm{E}$, and F, initially comprised a crystalline nano-sized B (97\% purity; SMI), pre-doped with 2 at.\% C, and mixed with $\mathrm{Mg}$ powder, with an Mg-to-B ratio of 1:2. All wires were formed to a diameter of $0.83 \mathrm{~mm}$, attaining a fill factor of $15 \%$. Then, all $\mathrm{MgB}_{2}$ wires were HIP processed at the Institute of High Pressure Physics in Warsaw [20]. All samples were annealed in a high-pressure gas chamber with $5 \mathrm{~N}$ argon atmosphere. The HIP process consisted of two steps: first, the isostatic pressure was applied, and subsequently, the temperature was ramped up to the annealing temperature [8]. At the end of the process, the annealing temperature was decreased to room temperature before decreasing the isostatic pressure. For these samples, the HIP process was performed at $570{ }^{\circ} \mathrm{C}$ at pressures of either $0.1 \mathrm{MPa}$ or $1.1 \mathrm{GPa}$ (Table 1).

The critical magnetic fields $\left(B_{\mathrm{c} 2}\right.$ and $\left.B_{\text {irr }}\right)$ and critical temperature $\left(T_{\mathrm{c}}\right)$ were measured by using a Physical Property Measurement System (PPMS), operating at $100 \mathrm{~mA}$ and $15 \mathrm{~Hz}$ at the Institute of Low Temperature and Structure Research (PAS) in Wroclaw. The values of $B_{\text {irr }}, T_{\mathrm{c}}$, and $B_{\mathrm{c} 2}$ were obtained by 10,50 , and $90 \%$ of normal-state resistivity criterion, respectively. The critical current $\left(I_{\mathrm{c}}\right)$ measurements were performed by the four-probe resistive method at liquid helium temperature in perpendicular magnetic field at the Institute of Low Temperature and Structure 
Table 1 The HIP process parameters and details of specifications of $\mathrm{MgB}_{2}$ samples in this study

\begin{tabular}{llllll}
\hline Sample identifier & Annealing time (min) & Temperature $\left({ }^{\circ} \mathrm{C}\right)$ & Pressure $(\mathrm{Pa})$ & Addition & B source \\
\hline A & 120 & 570 & $0.1 \mathrm{M}$ & $5 \mathrm{wt} \% \mathrm{C}_{4} \mathrm{H}_{6} \mathrm{O}_{5}$ & Tangshan \\
$\mathrm{B}$ & 120 & 570 & $1.1 \mathrm{G}$ & $5 \mathrm{wt} \% \mathrm{C}_{4} \mathrm{H}_{6} \mathrm{O}_{5}$ & Tangshan \\
$\mathrm{C}$ & 210 & 570 & $1.1 \mathrm{G}$ & $5 \mathrm{wt} \% \mathrm{C}_{4} \mathrm{H}_{6} \mathrm{O}_{5}$ & Tangshan \\
$\mathrm{D}$ & 120 & 570 & $0.1 \mathrm{M}$ & $2 \mathrm{wt} \% \mathrm{C}$ & SMI \\
E & 120 & 570 & $1.1 \mathrm{G}$ & $2 \mathrm{wt} \% \mathrm{C}$ & SMI \\
F & 210 & 570 & $1.1 \mathrm{G}$ & $2 \mathrm{wt} \% \mathrm{C}$ & SMI \\
\hline
\end{tabular}

Research (PAS) [16]. The value of the critical current was determined by the electric field criterion of $1 \mu \mathrm{V} / \mathrm{cm}$. An analysis of the wire's microstructure was carried out with a scanning electron microscope (FEI Nova Nano SEM 230 at the Institute of Low Temperature and Structure Research in Wroclaw).

\section{Results and Discussion}

The results in Fig. 1a, b for $\mathrm{C}_{4} \mathrm{H}_{6} \mathrm{O}_{5}$-doped $\mathrm{MgB}_{2}$ wires show that an increase of pressure from $0.1 \mathrm{MPa}$ to $1.1 \mathrm{GPa}$ yields smaller voids and a layered structure. In Fig. 1c, d, we see that higher annealing pressure $(1.1 \mathrm{GPa})$ leads to much smaller grains and voids and increased density of $\mathrm{MgB}_{2}$ material. Annealing in low pressure $(0.1 \mathrm{MPa})$ creates large voids with a size of about $5 \mu \mathrm{m}$. In contrast, high pressure $(1.1 \mathrm{GPa})$ forms voids of about $2 \mu \mathrm{m}$. These results suggest that annealing under a high pressure $(1.1 \mathrm{GPa})$ creates more connections between grains than that with a low pressure (0.1 MPa).

The SEM analysis (Fig. 2a, b), performed for the longitudinal section, shows that a longer annealing time (210 min) in high pressure does not have a significant influence on the size of voids and thickness of layers. Results in Fig. 2c, $\mathrm{d}$ indicate that the longer annealing time in high pressure $(1.1 \mathrm{GPa})$ slightly increases the size of grains and voids (from $2 \mu \mathrm{m}$ for $120 \mathrm{~min}$ to $3 \mu \mathrm{m}$ for $210 \mathrm{~min}$ ). It shows that
Fig. 1 The SEM images. Longitudinal sections of $\mathrm{C}_{4} \mathrm{H}_{6} \mathrm{O}_{5}$-doped $\mathrm{MgB}_{2}$ wires after the HIP process at a $0.1 \mathrm{MPa}$ and b $1.1 \mathrm{GPa}$ $\left(570{ }^{\circ} \mathrm{C}\right.$ for $\left.120 \mathrm{~min}\right)$. Cross sections of these samples after the HIP process at $\mathbf{c} 0.1 \mathrm{MPa}$ and d $1.1 \mathrm{GPa}\left(570{ }^{\circ} \mathrm{C}\right.$ for $\left.120 \mathrm{~min}\right)$
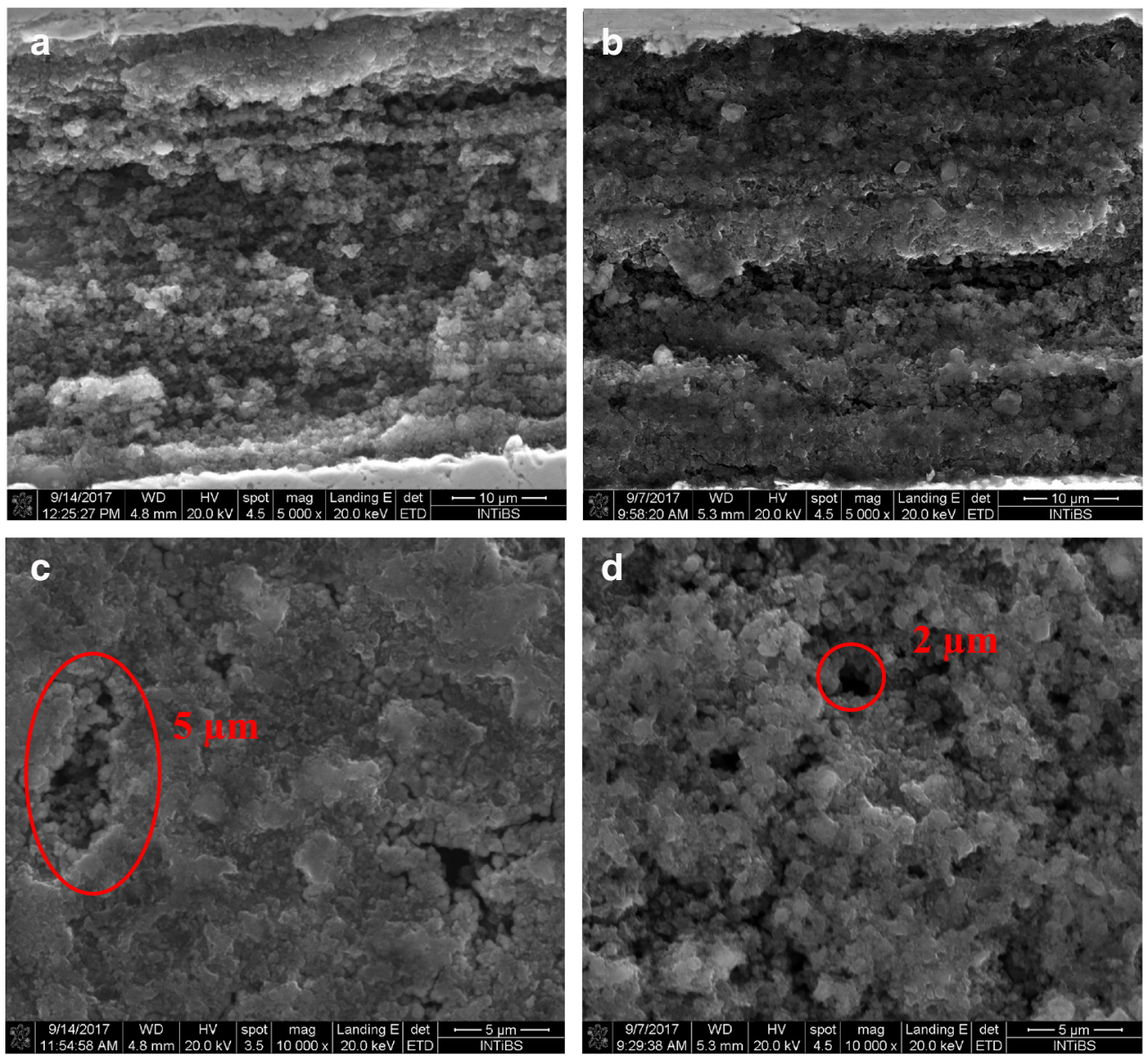
Fig. 2 The SEM images. Longitudinal sections of $\mathrm{C}_{4} \mathrm{H}_{6} \mathrm{O}_{5}$-doped $\mathrm{MgB}_{2}$ wires after the HIP process at $570{ }^{\circ} \mathrm{C}$ and $1.1 \mathrm{GPa}$ for a $120 \mathrm{~min}$ and b 210 min. Cross sections of these samples after the HIP process at $570{ }^{\circ} \mathrm{C}$ and $1.1 \mathrm{GPa}$ for $\mathbf{c} 120 \mathrm{~min}$ and $\mathbf{d} 210 \mathrm{~min}$
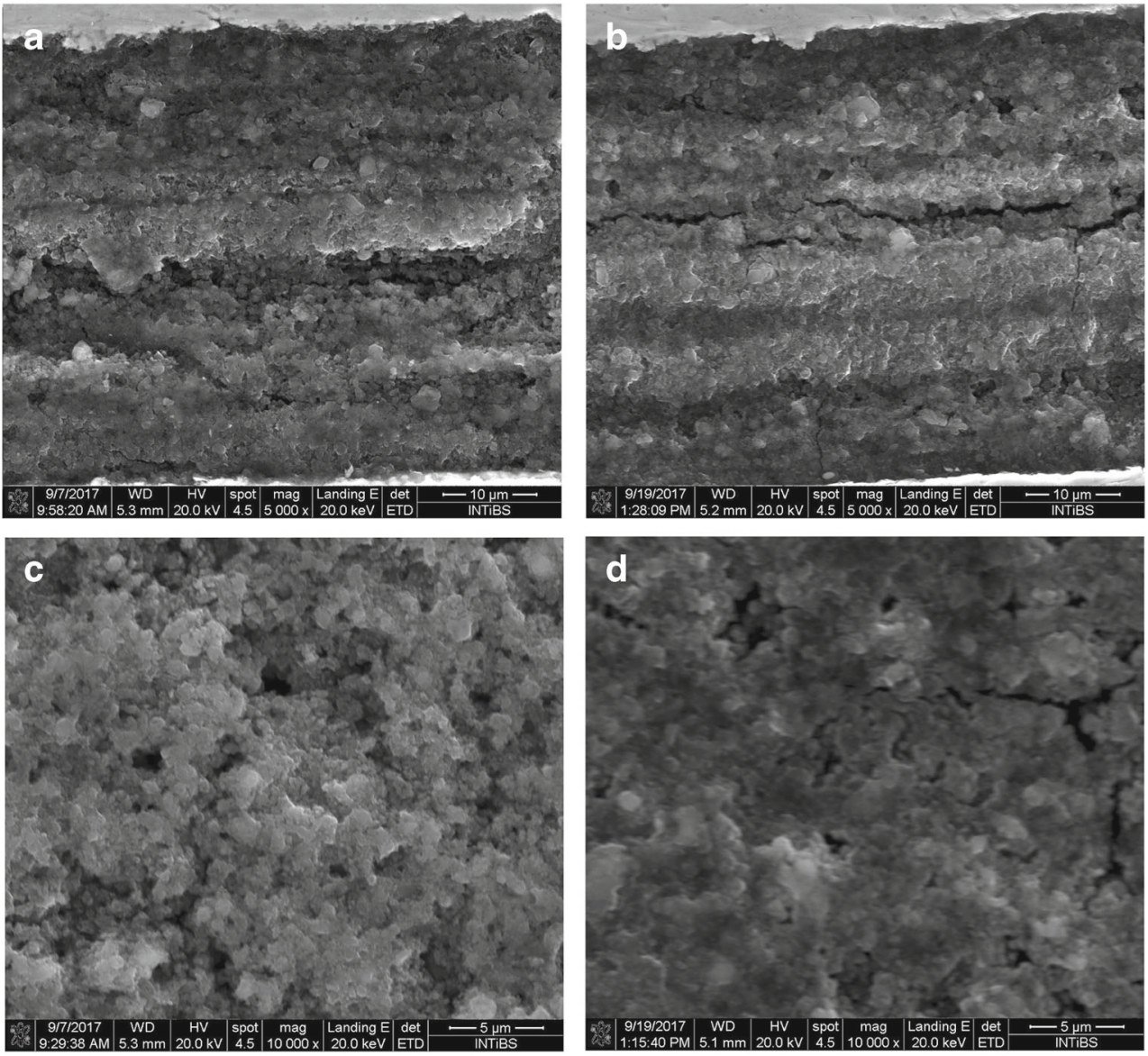

the densification process is finished already in $120 \mathrm{~min}$ of this annealing.

The SEM analysis (Fig. 3a, b), performed for C-doped $\mathrm{MgB}_{2}$ wires, shows that annealing under high pressure creates small voids and layered structure and increases the density of the $\mathrm{MgB}_{2}$ material and the number of intergrain connections. In Fig. 3d, we see that annealing in low pressure $(0.1 \mathrm{MPa})$ creates voids of a size of about $4 \mu \mathrm{m}$. In contrast, high pressure $(1.1 \mathrm{GPa})$ leads to voids of about $2 \mu \mathrm{m}$ (Fig. 3e). Figure 3c shows that longer annealing time (e.g., $210 \mathrm{~min}$, at $1.1 \mathrm{GPa}$ ) significantly increases the size of the voids $(10 \mu \mathrm{m})$. This leads to a significant reduction in the number of inter-grain connections.

In Figs. 1, 2, and 3, we see that the voids in samples A (0.1 $\mathrm{MPa}$, large boron, $\left.5 \mathrm{wt} \% \mathrm{C}_{4} \mathrm{H}_{6} \mathrm{O}_{5}\right)$ and $\mathrm{D}(0.1 \mathrm{MPa}$, small boron, $2 \mathrm{wt} \% \mathrm{C}$ ) both have similar size and, similarly, for samples B (1.1 GPa, large boron, $\left.5 \mathrm{wt} \% \mathrm{C}_{4} \mathrm{H}_{6} \mathrm{O}_{5}\right)$ and $\mathrm{E}(1.1 \mathrm{GPa}$, small boron, $2 \mathrm{wt} \% \mathrm{C})$. On the other hand, the SEM results for samples C $(1.1 \mathrm{GPa}$, large boron, $5 \mathrm{wt} \%$ $\left.\mathrm{C}_{4} \mathrm{H}_{6} \mathrm{O}_{5}\right)$ and $\mathrm{F}(1.1 \mathrm{GPa}$, small boron, $2 \mathrm{wt} \% \mathrm{C})$ show that the longer annealing time $(210 \mathrm{~min})$ for sample $\mathrm{F}$ leads to larger voids $(10 \mu \mathrm{m})$ than that for sample $\mathrm{C}(3 \mu \mathrm{m})$. Similarity in the size of voids in samples A and D and in samples B and E may suggest that the synthesis reaction in these samples has a similar rate. On the other hand, the larger size of voids in sample $\mathrm{F}$ indicates that smaller grains of crystalline boron, longer annealing time, and high pressure significantly accelerate the reaction rate. The results also indicate that high pressure slowly accelerates the reaction rate even in in situ $\mathrm{MgB}_{2}$ wires with large crystalline boron. Our results and Kim et al. [9] indicate that the crystalline boron reacts more slowly with $\mathrm{Mg}$ than the amorphous boron. The results for samples $\mathrm{A}-\mathrm{F}$ also show that $\mathrm{C}_{4} \mathrm{H}_{6} \mathrm{O}_{5}$ admixture does not influence the rate of reaction at $570{ }^{\circ} \mathrm{C}$ although it has a low melting point of about $130-150{ }^{\circ} \mathrm{C}$. This may also suggest that the admixture has a slight influence on the structure of in situ $\mathrm{MgB}_{2}$ wires at an annealing temperature of $570{ }^{\circ} \mathrm{C}$. Our results and Kim et al. suggest that $\mathrm{C}$ slowly substitutes for B at a low annealing temperature [9].

Kim et al. showed that crystalline nano-boron (SMI) has about four times more defects than large crystalline boron (Tangshan) [9]. Kittel indicated that density of dislocations (shift dislocations) had an influence on the rate of reactions in materials [26]. The SEM images for samples A (0.1 MPa; Tangshan), D (0.1 MPa; SMI), B (1.1 GPa; Tangshan), and E (1.1 GPa; SMI) show that these samples have similar 

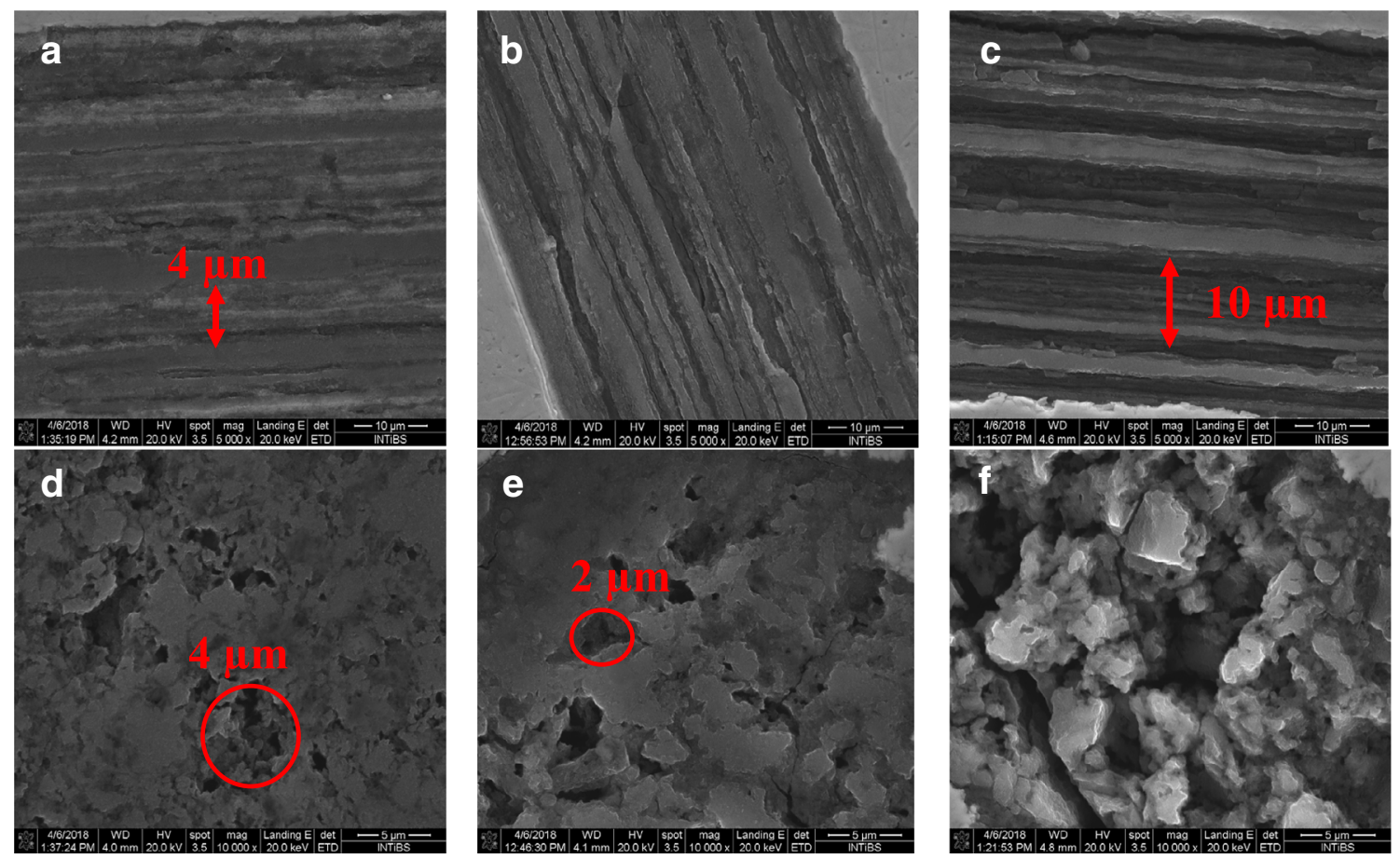

Fig. 3 The SEM images. Longitudinal sections of C-doped $\mathrm{MgB}_{2}$ wires after the $\mathrm{HIP}$ process at $570{ }^{\circ} \mathrm{C}$ and a $0.1 \mathrm{MPa}$ for $120 \mathrm{~min}$, b $1.1 \mathrm{GPa}$ for $120 \mathrm{~min}$, and c $1.1 \mathrm{GPa}$ for $210 \mathrm{~min}$. Cross sections

structure (size of voids is similar) after annealing time by $120 \mathrm{~min}$. This also might indicate that the size of the crystalline boron grains has a weak influence on the rate of reaction of $\mathrm{Mg}$ with $\mathrm{B}$ at low annealing temperature. The low impact of the boron grain size on the rate of the reaction can be related to the higher hardness of the crystalline boron. The results of samples B and C (Tangshan) show that an increase of the annealing time from 120 to $210 \mathrm{~min}$ does not increase the size of voids (Fig. 2). On the other hand, the SEM results for sample F (SMI) show that longer annealing time does form large voids. This indicates that the higher number of defects, high pressure, and long annealing time increase the rate of the reaction in $\mathrm{MgB}_{2}$ material with crystalline nano-boron. In addition, the results show that the smaller number of defects, high pressure, and long annealing time do not accelerate the rate of synthesis reaction in $\mathrm{MgB}_{2}$ material with large crystalline boron (Tangshan). These results may also suggest that high pressure during annealing at low temperature needs a longer annealing time to activate defects in nano-crystalline boron, which leads to the reaction of $\mathrm{Mg}$ and $\mathrm{B}$.

The transport measurements show that an increase of pressure from $0.1 \mathrm{MPa}$ to $1.1 \mathrm{GPa}$ decreases the $T_{\mathrm{c}}(B=$ $0 \mathrm{~T}$ ) by about $2 \mathrm{~K}\left(T_{\mathrm{c}}\right.$ of 34 and $32 \mathrm{~K}$ for samples $\mathrm{A}$ and $\mathrm{B}$, respectively). Monteverde et al. show that an increase of pressure from 0 to $25 \mathrm{GPa}$ causes $T_{\mathrm{c}}$ to decrease from 39 of these samples after the HIP process at $570{ }^{\circ} \mathrm{C}$ and $\mathbf{d} 0.1 \mathrm{MPa}$ for $120 \mathrm{~min}$, e $1.1 \mathrm{GPa}$ for $120 \mathrm{~min}$, and $\mathbf{f} 1.1 \mathrm{GPa}$ for $210 \mathrm{~min}$

to $20 \mathrm{~K}$ [27]. They indicate that $T_{\mathrm{c}}$ can reduce defects in the crystal lattice and change the concentration of carriers [27]. Similar results were obtained for in situ $\mathrm{MgB}_{2}$ wires with amorphous boron $\left(10 \% \mathrm{SiC}\right.$ admixture, $700{ }^{\circ} \mathrm{C}$, and $1 \mathrm{GPa}$ decrease the $T_{\mathrm{c}}$ by about $3 \mathrm{~K}$ ) [19]. Earlier, our results indicated that a decrease of $T_{\mathrm{c}}$ was caused by the crystal dislocations created by the HIP process. In Fig. 4a, we see that high pressure significantly increases $B_{\text {irr }}$ in the range from 3 to $22 \mathrm{~K}$ and reduces $B_{\text {irr }}$ above $22 \mathrm{~K}$. Similar results were obtained for in situ $\mathrm{MgB}_{2}$ wires made with amorphous boron [28]. The $B_{\text {irr }}$ value is dependent on the high-field pinning centers e.g., dislocations, vacancies, and substitutions to the crystal lattice. Serquis et al. [17] pointed out that the HIP process increases the density of dislocations. Kim et al. [9] and SEM images (Figs. 1, 2, and 3) indicate that $\mathrm{C}$ slowly substitutes for B during annealing at low temperature. This indicates that high pressure mainly increases the density of high-field pinning centers (dislocations) which increases $B_{\text {irr }}$ in the range from 3 to $22 \mathrm{~K}$. On the other hand, high pressure decreases $B_{\text {irr }}$ above $22 \mathrm{~K}$. This also indicates that dislocations are strong pinning centers at low and medium temperatures but weaker pinning centers at high temperature. Kwok et al. also indicate that irradiation defects (similar to dislocations) only improve pinning at high magnetic fields and not at very high temperatures [29]. The weaker anchoring of the vortex lattice by 

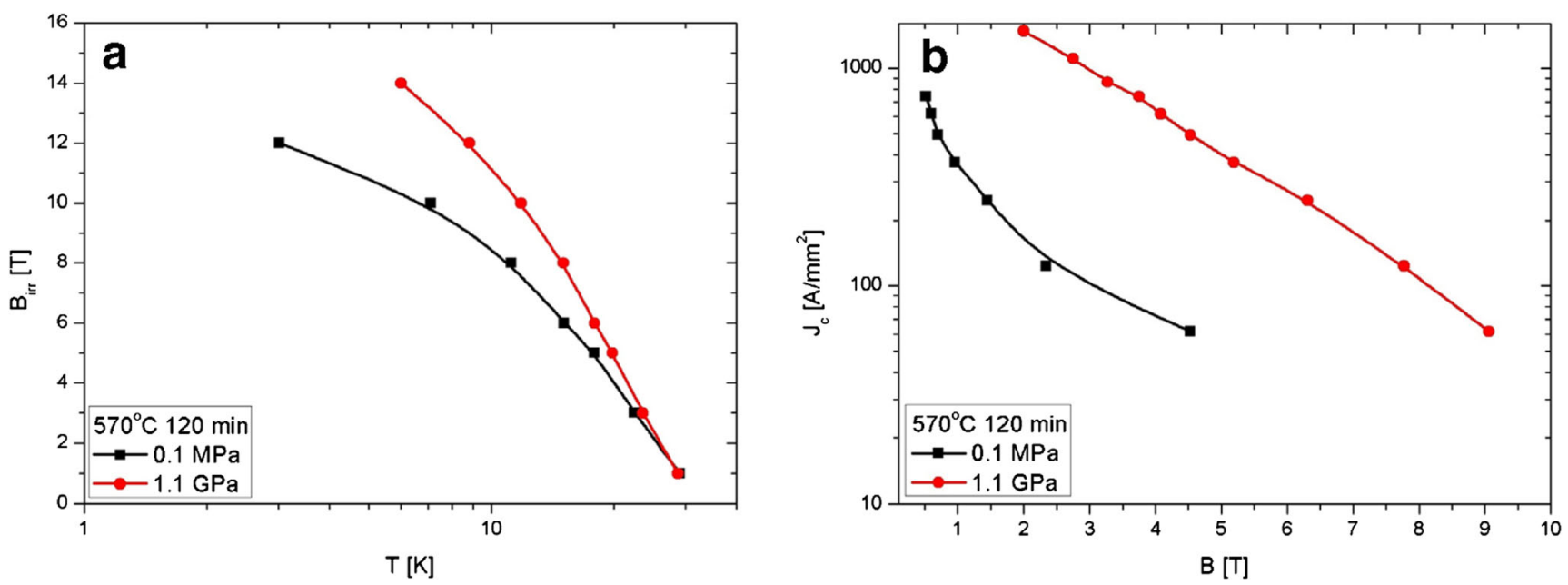

Fig. 4 Transport measurements for samples A and B. a The irreversibility magnetic field dependence on the temperature. $\mathbf{b}$ The critical current density as a function of magnetic field at $4.2 \mathrm{~K}$

dislocations and defects created by irradiation at high temperature is caused by these defects, decreasing the $T_{\mathrm{c}}$ of all superconducting materials.

The measurements in Fig. $4 \mathrm{~b}$ show that annealing in high pressure increases $J_{\mathrm{c}}$ of in situ $\mathrm{MgB}_{2}$ wires ninefold in low, middle, and high magnetic fields. Research indicates that the simultaneous increase in the density of pinning centers and connections between the grains would significantly increase $J_{\mathrm{c}}$ in in situ $\mathrm{MgB}_{2}$ wires [19]. This indicates that high pressure $(1.1 \mathrm{GPa})$ and low annealing temperature $\left(570{ }^{\circ} \mathrm{C}\right)$ lead to high density of low-, middle-, and high-field pinning centers and increase the number of connections between the grains. The SEM images indicate that voids may be responsible for creation of low and middle-field pinning centers [3]. The $B_{\text {irr }}$ results in Fig. 4a suggest that the $\mathrm{C}_{4} \mathrm{H}_{6} \mathrm{O}_{5}$ admixture mainly forms surface and point pinning centers. Additionally, the $B_{\text {irr }}$ results show that high-field pinning centers (here, dislocations) are mainly created by the HIP process in sample B.

The transport measurements show that the increase of the annealing time from 120 to $210 \mathrm{~min}$ does not change $T_{\mathrm{c}}$ $\left(B=0 \mathrm{~T}\right.$ ) but slightly decreases $B_{\text {irr }}$ below $9 \mathrm{~K}$ (Fig. 5a). This small reduction of $B_{\text {irr }}$ indicates that longer annealing time slightly reduces the density of high-field pinning centers. The measurements (Fig. 5b) show that the increase of the annealing time from 120 to 210 min leads to a reduction of $J_{\mathrm{c}}$ by about $10 \%$ at $4.2 \mathrm{~K}$. Hence, high pressure, low annealing temperature, and long annealing time $(210 \mathrm{~min})$ only slightly change $B_{\text {irr }}$ and $J_{\mathrm{c}}$ in high magnetic fields. This indicates that substitution of $\mathrm{C}$ for $\mathrm{B}$ is very slow. Even high

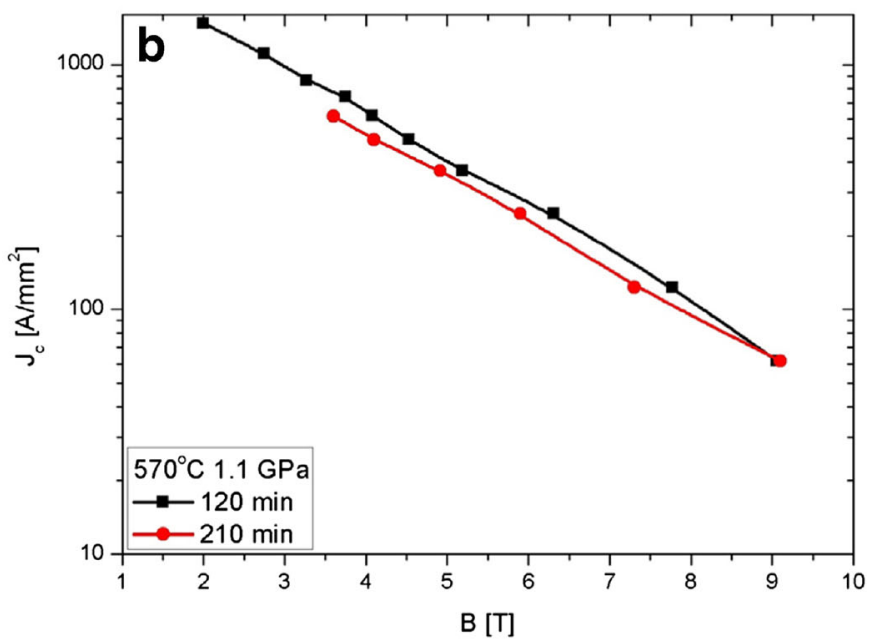

Fig. 5 Transport measurements for samples B and C. a The irreversibility magnetic field dependence on the temperature. $\mathbf{b}$ The critical current density as a function of magnetic field at $4.2 \mathrm{~K}$ 
pressure and an admixture with a low-melting point dopant do not accelerate the rate of substitution of $\mathrm{C}$ for $\mathrm{B}$ comprising of large crystalline boron grains. Similar results were obtained by Kim et al. [9]. They showed that a long annealing time (240 $\mathrm{min})$ and a lower annealing temperature of $600{ }^{\circ} \mathrm{C}$ did not increase $B_{\text {irr }}$ and $J_{\mathrm{c}}$ in $10 \mathrm{wt} \%$ $\mathrm{C}_{4} \mathrm{H}_{6} \mathrm{O}_{5}$-doped in situ $\mathrm{MgB}_{2}$ wires [9].

Transport measurements performed for $2 \% \mathrm{C}$-doped in situ $\mathrm{MgB}_{2}$ wires with nano-crystalline boron (SMI) show that annealing in high pressure decreases critical temperature $\left(T_{\mathrm{c}}\right)$ by about $2 \mathrm{~K}$ (sample $\mathrm{A}: T_{\mathrm{c}}=34 \mathrm{~K}$, sample B: $T_{\mathrm{c}}=32 \mathrm{~K}$ ), significantly increases $B_{\text {irr }}$ (Fig. 6a) in the range from 5 to $22 \mathrm{~K}$, and decreases $B_{\text {irr }}$ above $22 \mathrm{~K}$. This also indicates that high-field pinning centers are strong pinning centers at low and middle temperatures and weak pinning centers at temperatures higher than $22 \mathrm{~K}$. The results above indicate that high pressure significantly increases the density of the high-field pinning centers in 2\% C-doped in situ $\mathrm{MgB}_{2}$ wires with nano-crystalline boron. Based on the results above, we can conclude that high pressure mainly forms high-field pinning centers. The results in Fig. 6a show that the long annealing time (sample F, $210 \mathrm{~min}$ ) and high pressure significantly reduce $B_{\text {irr }}$ in the range from 7 to $22 \mathrm{~K}$ and do not change $B_{\text {irr }}$ above $22 \mathrm{~K}$. This shows that long annealing time significantly reduces the density of high-field pinning centers. The value of $B_{\text {irr }}$ depends on the high-field pinning centers. Kittel [26] indicated that dislocations always occur in the structure of materials with higher or lower density. The SEM images (Fig. 3f) show that sample $\mathrm{F}$ has a large amount of big voids. Big voids reduce the number of connections between the grains. High-field pinning centers are located at the grains and on connections

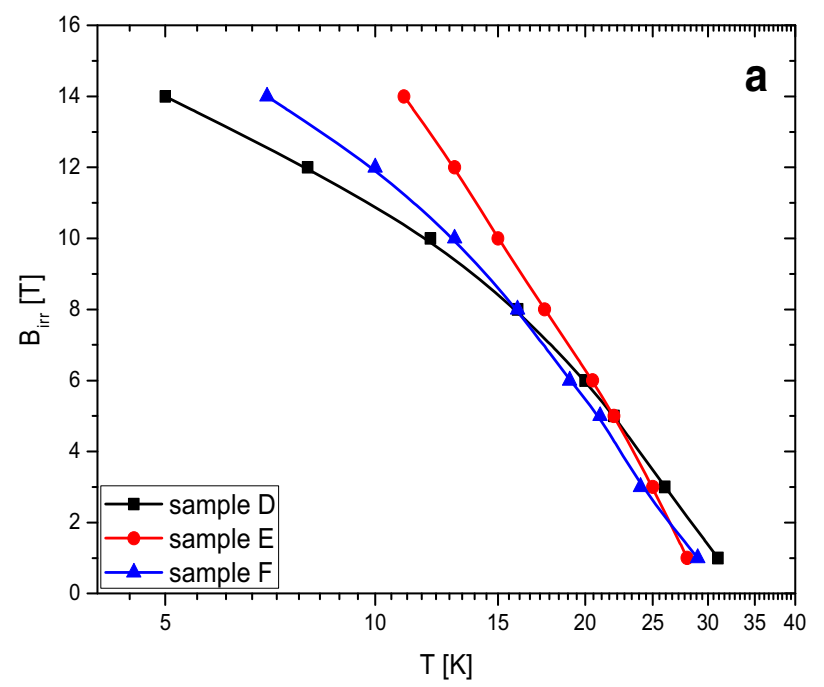

between grains. This leads to a reduction in the density of high-field pinning centers and $B_{\text {irr. }}$. Another reason may be the change in the dominant pinning mechanism. Figure $6 \mathrm{~b}$ shows that annealing in high pressure increases $J_{\mathrm{c}}$ in doped in situ $\mathrm{MgB}_{2}$ wire, almost by 2 orders of magnitude in magnetic fields from 8 to $14 \mathrm{~T}$ (sample $\mathrm{D}, 20 \mathrm{~A} / \mathrm{mm}^{2}$ in 7.2 T; sample E, $1000 \mathrm{~A} / \mathrm{mm}^{2}$ in $7.2 \mathrm{~T}$ ). This indicates that high pressure and annealing time of $120 \mathrm{~min}$ significantly increase the density of high-field pinning centers and the connections between the grains. Longer annealing time (sample F, $210 \mathrm{~min}$ ) in high pressure causes reduction of $J_{\mathrm{c}}$ in all magnetic fields. SEM images (Fig. 3c) show that sample $\mathrm{F}$ has more surface and point pinning centers (voids) and significantly less connections between grains than sample E (120 min and 1.1 GPa). Surface and point pinning centers increase $J_{\mathrm{c}}$ in low and middle magnetic fields. However, sample $\mathrm{F}$ has relatively low $J_{\mathrm{c}}$ in low magnetic fields. This indicates that the high density of pinning centers and the small number of connections between grains do not lead to an increase in $J_{\mathrm{c}}$. High-field pinning centers are found inside the grains and in the borders of connections and between the grains. Therefore, the reduction of the number of connections between the grains in sample $F$ causes a significant reduction in $J_{\mathrm{c}}$ in high magnetic field. This indicates that high-field pinning centers are present in the connections between the grains where the transport current is carried. The high $J_{c}$ in the high magnetic fields in sample $E$ indicates that the high pressure creates a large number of connections between grains and grains with high density of high-field pinning centers.

Pelissier showed that high pressure increases the melting point of $\mathrm{Mg}$ and reduces the rate of $\mathrm{C}$ substitution for B [30].

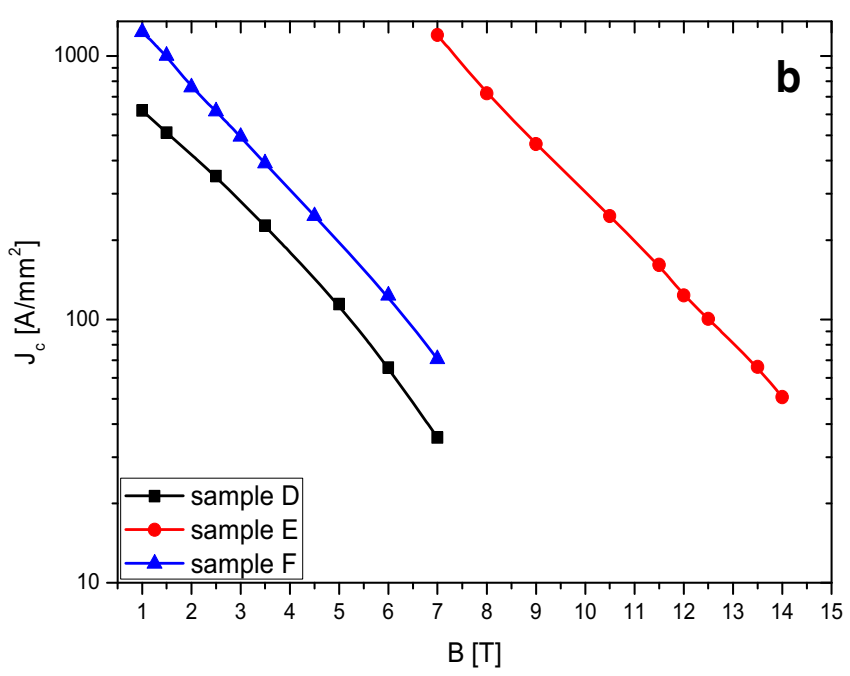

Fig. 6 Transport measurements for samples D, E, and F. a The irreversibility magnetic field dependence on the temperature. b The critical current density as a function of magnetic field at $4.2 \mathrm{~K}$ 
The SEM results indicate that substantial C substitution for $\mathrm{B}$ is only observed in sample $\mathrm{F}$. The above factors indicate that there is little substitution of $\mathrm{C}$ for $\mathrm{B}$ in samples $\mathrm{D}$ and E. This indicates that $J_{\mathrm{c}}$ and $B_{\text {irr }}$ in samples $\mathrm{D}$ and $\mathrm{E}$ are mainly dependent on the size of the crystalline boron grains, pressure, and defects inside the crystalline boron grains.

Previous research on NbTi showed that high-field pinning centers can be created by three types of defects inside the grains, namely dislocations, substitution for the crystal lattice, and inside precipitation [1]. These defects anchor a vortex lattice in various ranges of high magnetic fields. Jung et al. indicate that $\mathrm{MgB}_{2}$ material shrinks up to $25 \%$ by volume during the synthesis reaction, leading to the formation of voids and strains [5]. Comparing the results of samples A and D (Fig. 7), we see that these samples have the same critical temperature $(34 \mathrm{~K})$ and $B_{\text {irr }}=f(T)$ has the same shape. Sample D has a higher $B_{\text {irr }}$ by $15 \%$ than sample A. Samples A and D were heat treated at a low pressure of about $0.1 \mathrm{MPa}$. This indicates that the strains are mainly due to $\mathrm{Mg}$ shrinkage. The higher $B_{\text {irr }}$ in sample D indicates that the nano-crystalline boron with more defects creates more strains than the large crystalline boron with fewer defects (sample A). Heat treatment at a high pressure of 1.1 GPa increases the density of dislocations in samples B and E (Fig. 7). The results (Fig. 7) show that high pressure significantly increases the $B_{\text {irr }}$ value at low and medium temperatures and decreases $B_{\text {irr }}$ at high temperatures in samples with nano-crystalline and large crystalline boron. This shows that the HIP process increases very effectively the density of dislocations in in situ $\mathrm{MgB}_{2}$ wires. This also indicates that dislocations are good in both high-field pinning centers and low- and middle-temperature pinning centers. In addition, the results also indicate that small grains of crystalline boron with a high density of defects and high

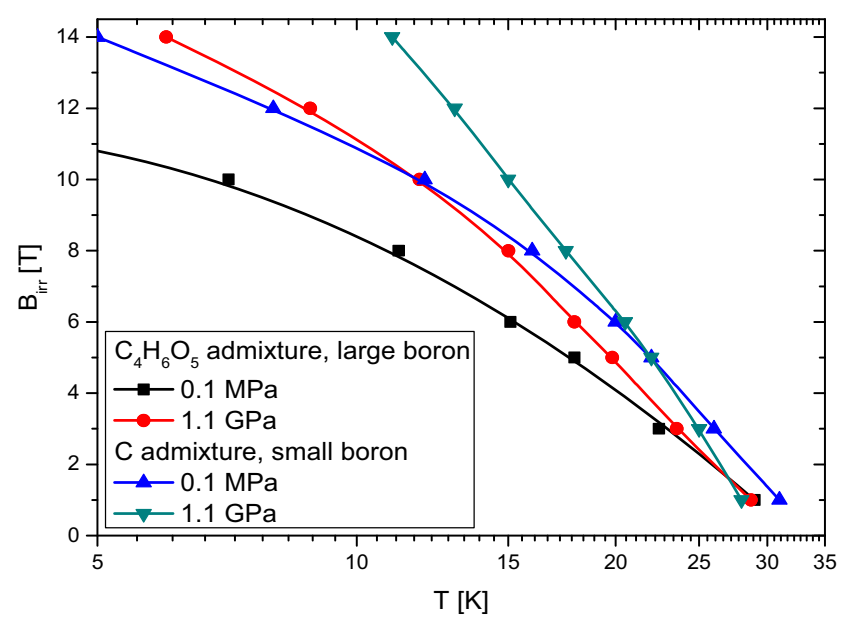

Fig. 7 The irreversibility magnetic field dependence on the temperature for samples A, B, D, and E pressure allow for a very significant increase of $B_{\text {irr }}$ in in situ $\mathrm{MgB}_{2}$ wires.

Comparing the results of samples B and D (Fig. 7), we see that the temperature dependence characteristics of $B_{\text {irr }}$ of these samples have a different shape. This indicates another type of dominant pinning centers in sample D. In sample D (0.1 MPa), Mg shrinkage mainly forms highfield pinning centers due to strains. On the other hand, high pressure in sample B mainly creates high-field pinning centers due to dislocations. Measurements indicate that large grains of crystalline boron create a smaller amount of strains (sample A) than small grains of crystalline boron. Sample E (1.1 GPa and small grains) has a lot of dislocations and strains. This makes it difficult to analyze its pinning centers. Based on the results for samples B and D, we can conclude that dislocations are more effective in increasing $B_{\text {irr }}$ below $12 \mathrm{~K}$ but only slightly increase $B_{\text {irr }}$ above $12 \mathrm{~K}$. The results also indicate that strains more effectively increase $B_{\text {irr }}$ above $12 \mathrm{~K}$ and less below $12 \mathrm{~K}$. Results for sample $\mathrm{E}$ are very encouraging, because these show that small grains of crystalline boron and high isostatic pressure result in a large amount of strains and dislocations, which significantly increase $B_{\text {irr }}$ in low and middle temperatures. The results in Fig. 7 indicate that the dislocations more strongly reduce $T_{\mathrm{c}}$ in in situ $\mathrm{MgB}_{2}$ wires than those of the strains due to $\mathrm{Mg}$ shrinkage.

\section{Conclusions}

This research shows that a high isostatic pressure of $1.1 \mathrm{GPa}$ at low annealing temperature $\left(570{ }^{\circ} \mathrm{C}\right)$ leads to significant increase in the density of high-field pinning centers in in situ $\mathrm{MgB}_{2}$ wires made with both small- and large-sized crystalline boron. Furthermore, transport measurements show that an increase of annealing time from 120 to $210 \mathrm{~min}$ significantly reduces $J_{\mathrm{c}}$ and $B_{\text {irr }}$ only for $\mathrm{MgB}_{2}$ wires with small crystalline boron, indicating that longer annealing time significantly reduces the number of high-field pinning centers. The results show that dislocations created by the HIP process significantly increase $B_{\text {irr }}$ in the temperature range from 5 to $12 \mathrm{~K}$. On the other hand, strains created by shrinkage of $\mathrm{Mg}$ increase $B_{\text {irr }}$ in the temperature range from 12 to $34 \mathrm{~K}$. The reduction of $T_{\mathrm{c}}$ in in situ $\mathrm{MgB}_{2}$ wires is more strongly influenced from dislocations than that of the strains created due to $\mathrm{Mg}$ shrinkage. In addition, the results indicate that the rate of reaction is more dependent on the size of crystalline boron grains and defects than the type of admixture used. The studies indicate that high pressures may accelerate the reaction rate for in situ $\mathrm{MgB}_{2}$ wires, where small boron crystals contain a high density of defects. The obtained results are very important because they show 
that small crystalline boron and high isostatic pressure allow a large amount of strains and dislocations to develop, which significantly increase $B_{\text {irr }}$ in temperatures up to $25 \mathrm{~K}$.

Open Access This article is distributed under the terms of the Creative Commons Attribution 4.0 International License (http:// creativecommons.org/licenses/by/4.0/), which permits unrestricted use, distribution, and reproduction in any medium, provided you give appropriate credit to the original author(s) and the source, provide a link to the Creative Commons license, and indicate if changes were made.

\section{References}

1. Gajda, D., Zaleski, A.J., Morawski, A., Hossain, M.S.A.: Supercond. Sci. Technol. 30, 085011 (2017)

2. Flukiger, R., Hossain, M.S.A., Senatore, C.: Supercond. Sci. Technol. 22, 085002 (2009)

3. Livingston, J.D.: GE R\&D Center Report (1969-1970), see www. bnl.gov/magnets/staff/gupta/Summer1968/0377.pdf

4. Gajda, D., Morawski, A., Zaleski, A.J., Akdoğan, M., Yetiş, H., Karaboğa, F., Cetner, T., Belenli, İ.: J. Supercond. Novel Magn. 30, 3397-3402 (2017)

5. Jung, A., Schlachter, S.I., Runtsch, B., Ringsdorf, B., Fillinger, H., Orschulko, H., Drechsler, A., Goldacker, W.: Supercond. Sci. Technol. 23, 095006 (2010)

6. Kazakov, S.M., Puzniak, R., Rogacki, K., Mironov, A.V., Zhigadlo, N.D., Jun, J., Soltmann, C., Batlogg, B., Karpinski, J.: Phys. Rev. B 71, 024533 (2005)

7. Buzea, C., Yamashita, T.: Supercond. Sci. Technol. 14, 115-146 (2001)

8. Gajda, D., Morawski, A., Zaleski, A.J., Häßler, W., Nenkov, K., Małecka, M., Rindfleisch, M.A., Hossain, M.S.A., Tomsic, M.: J. Appl. Phys. 120, 113901 (2016)

9. Kim, J.H., Heo, Y., Matsumoto, A., Kumakura, H., Rindfleisch, M., Tomsic, M., Dou, S.X.: Supercond. Sci. Technol. 23, 075014 (2010)

10. Mustapić, M., Horvat, J., Hossain, M.S., Sun, Z., Skoko, Z., Mitchell, D.R.G., Dou, S.X.: Acta. Mater. 70, 298 (2014)

11. Bovone, G., Matera, D., Bernini, C., Magi, E., Vignolo, M.: Supercond. Sci. Technol. 28, 065006 (2015)
12. Kim, J.H., Zhou, S., Hossain, M.S.A., Pan, A.V., Dou, S.X.: Appl. Phys. Lett. 89, 142505 (2006)

13. Zhang, X., Wang, D., Gao, Z., Wang, L., Qi, Y., Zhang, Z., Ma, Y., Awaji, S., Nishijima, G., Watanabe, K., Mossang, E., Chaud, X.: Supercond. Sci. Technol. 23, 025024 (2010)

14. Gajda, D., Morawski, A., Zaleski, A.J., Cetner, T., Małecka, M., Presz, A., Rindfleisch, M., Tomsic, M., Thong, C.J., Surdacki, P.: Supercond. Sci. Technol. 26, 105008 (2013)

15. Gajda, D., Morawski, A., Zaleski, A., Kurnatowska, M., Cetner, T., Gajda, G., Presz, A., Rindfleisch, M.A., Tomsic, M.: Supercond. Sci. Technol. 28, 015002 (2015)

16. Gajda, D., Zaleski, A.J., Morawski, A., Rindfleisch, M., Thong, C., Tomsic, M.: Supercond. Sci. Technol. 28, 115003 (2015)

17. Serquis, A., Civale, L., Hammon, D.L., Liao, X.Z., Coulter, J.Y., Zhu, Y.T., Jaime, M., Peterson, D.E., Mueller, F.M., Nesterenko, V.F., Gu, Y.: Appl. Phys. Lett. 82, 2847-2849 (2003)

18. Liao, X.Z., Serquis, A., Zhu, Y.T., Civale, L., Hammon, D.L., Peterson, D.E., Mueller, F.M., Nesterenko, V.F., Gu, Y.: Supercond. Sci. Technol. 16, 799 (2003)

19. Gajda, D., Zaleski, A.J., Morawski, A., Rindfleisch, M.A., Thong, C., Tomsic, M.: Supercond. Sci. Technol. 29, 085010 (2016)

20. Adamczyk, K., Morawski, A., Cetner, T., Zaleski, A., Gajda, D., Rindfleisch, M., Tomsic, M., Diduszko, R., Presz, A.: IEEE Trans. Appl. Supercond. 22, 620 (2012)

21. Birajdar, B., Eibl, O.: J. Appl. Phys. 105, 033903 (2009)

22. Takahashi, M., Okada, M., Nakane, T., Kumakura, H.: Supercond. Sci. Technol. 22, 125017 (2009)

23. Gajda, D., Morawski, A., Zaleski, A.J., Akdoğan, M., Yetiş, H., Karaboğa, F., Cetner, T., Belenli, İ.: J. Alloys Compd. 687, 616622 (2016)

24. Maeda, M., Hossain, M.S., Motaman, A., Kim, J.H., Kario, A., Rindfleisch, M., Tomsic, M., Dou, S.X.: IEEE T. Appl. Supercond. 23(3), 6200704 (2013)

25. Tomsic, M., Rindflesich, M., Yue, J., McFadden, K., Phillips, J., Sumption, M.D., Bhatia, M., Bohnenstiehl, S., Collings, E.W.: Int. J. Appl. Ceram. Technol. 4, 250-259 (2007)

26. Kittel, C.: Introduction to Solid State Physics. Wiley, New York (2005)

27. Monteverde, M., Nunez-Regueiro, M., Rogado, N., Regan, K.A., Hayward, M.A., He, T., Loureiro, S.M., Cava, R.J.: Science 293, 75-77 (2001)

28. Gajda, D., Morawski, A., Zaleski, A.J., Häßler, W., Nenkov, K., Rindfleisch, M., Cetner, T., Tomsic, M.: J. Mater. Sci. Eng. 5, 1000244 (2016)

29. Kwok, W.K., Welp, U., Glatz, A., Koshelev, A.E., Kihistrom, K.J., Crabtree, G.W.: Rep. Prog. Phys. 79, 116501 (2016)

30. Pelissier, J.L.: Phys. Scr. 34, 838 (1986) 